inflammatory agonists to induce interleukin $6(p<0.05)$ and macrophage inflammatory protein $-1 \alpha(p<0.01)$ production. Ang-2 cooperatively induced monocyte chemotactic protein-1 production $(\mathrm{p}<0.05)$ and suppressed TNF- and LPS-dependent production of the cell adhesion inhibitor thrombospondin 2 (TSP2) by $50 \%(p<0.05)$.

Conclusion Engagement of macrophage Tie2 can enhance accumulation of white blood cells at the site of inflammation by sensitising macrophage production of chemokines and cytokines. In particular, Ang-2 suppression of TSP2 would enhance retention of infiltrating cells. Our results represent a novel mechanism by which angiogenic factors contribute to inflammation.

\section{A92 MACROPHAGE STIMULATION BY ANGIOPOIETINS-1 AND -2 PROMOTES RECRUITMENT AND RETENTION OF WHITE BLOOD CELLS AT SITES OF INFLAMMATION}

S Krausz, M E Sanders, P P Tak, K A Reedquist Division of Clinical Immunology and Rheumatology, Academic Medical Center, University of Amsterdam, The Netherlands

\subsection{6/ard.2010.129627e}

Background The authors have previously found that macrophages are predominant targets of the angiogenic factors angiopoietin (Ang)-1 and -2 in synovial tissue of patients with rheumatoid arthritis (RA). How macrophage Tie2 ligation by Ang might contribute to disease in RA is currently unknown. Objective To investigate the effects of Ang-1 and Ang-2 stimulation on the inflammatory activation of human macrophages.

Methods Peripheral blood-derived human macrophages were stimulated with increasing concentrations of Ang-1 and Ang-2 in the absence or presence of tumour necrosis factor (TNF) or lipopolysaccharide (LPS). Supernatants were collected and analysed for production of soluble mediators of angiogenesis and inflammation by proteomic and ELISA analysis.

Results Ang-1 and Ang-2 did not stimulate macrophages by themselves, but both synergistically cooperated with 